\title{
Conformal Triality of de Sitter, Minkowski and Anti-de Sitter Spaces *
}

\author{
Bin Zhou ${ }^{\dagger}$ \\ Department of Physics, Beijing Normal University, Beijing 100875, China \\ Han-Ying Guo $\ddagger$ \\ Institute of Theoretical Physics, Chinese Academy of Sciences \\ P.O. Box 2735, Beijing 100080, China
}

December 19, 2005

\begin{abstract}
We describe how conformal Minkowski, $d S$ - and $A d S$-spaces can be united into a single submanifold $[\mathcal{N}]$ of $\mathbb{R} P^{5}$. It is the set of generators of the null cone in $\mathcal{M}^{2,4}$. Conformal transformations on the Mink-, $d S$ - and $A d S$-spaces are induced by $O(2,4)$ linear transformations on $\mathcal{M}^{2,4}$. We also describe how Weyl transformations and conformal transformations can be resulted in on $[\mathcal{N}]$. In such a picture we give a description of how the conformal Mink-, $d S$ - and $A d S$-spaces as well as $[\mathcal{N}]$ are mapped from one to another by conformal maps. This implies that a CFT in one space can be translated into a CFT in another. As a consequence, the AdS/CFT-correspondence should be extended.
\end{abstract}

\section{Introduction}

In this talk we show how three kinds of spaces of constant curvatures are "unified" into a single space by conformal maps: the conformal Mink-, $d S$ - and $A d S$-spaces are the same nature, resulted in from a hypersurface $[\mathcal{N}]$ of $\mathbb{R} P^{5}$. Here $[\mathcal{N}]$ is the quotient space from the null "cone" $\mathcal{N}$ of $\mathcal{M}^{2,4}$ with the vertex at the origin. Although no metric on $[\mathcal{N}]$ can be induced naturally from $\mathcal{M}^{2,4}$, a set of metrics can be obtained, differing from each other by a Weyl factor. For a given metric on $[\mathcal{N}]$, an $O(2,4)$ linear transformation on $\mathcal{M}^{2,4}$ induces a conformal transformation.

Starting from this picture, it is not astonishing that the conformal Minkspace, $d S^{4}$ and $A d S^{4}$ can be conformally mapped from one to another. This

\footnotetext{
*This work is supported partly by NSFC (10505004, 10375087, 90503002).

†Email: zhoub@bnu.edu.cn

${ }^{\ddagger}$ Email: hyguo@itp.ac.cn
} 
technique can be used to translate the content of a CFT from one space to another. Thus, if we have the $A d S / C F T$ correspondence 1 between $A d S^{5}$ and the conformal Mink-space, then we also have various correspondences: $A d S^{5}$ between conformal $d S^{4}, A d S^{4}$ or $[\mathcal{N}]$.

\section{The Hypersurface $[\mathcal{N}] \subset \mathbb{R} P^{5}$}

\subsection{The $O(2,4)$-Invariant Hypersurface of $\mathbb{R} P^{5}$}

For the $(2+4)$-d Mink-space $\mathcal{M}^{2,4}$ endowed with the inner product

$$
\zeta_{1} \cdot \zeta_{2}:=\eta_{\hat{A} \hat{B}} \zeta_{1}^{\hat{A}} \zeta_{2}^{\hat{B}}, \quad\left(\eta_{\hat{A} \hat{B}}\right)=\operatorname{diag}(1,-1, \ldots,-1,1),
$$

where $\hat{A}, \hat{B}=0,1, \ldots, 5$, we consider its null cone

$$
\mathcal{N}: \quad \zeta \cdot \zeta=0, \quad(\zeta \neq 0) .
$$

In $\mathcal{M}^{2,4}$ there is the standard equivalence relation $\sim$, defined by

$$
\boldsymbol{\zeta}^{\prime} \sim \boldsymbol{\zeta} \Leftrightarrow \boldsymbol{\zeta}^{\prime}=c \boldsymbol{\zeta} \text { for a nonzero } c \in \mathbb{R}
$$

which makes the quotient space $\mathcal{M}^{2,4}-\{0\} / \sim$ to be the projective space $\mathbb{R} P^{5}$. The equivalence class of a nonzero $\boldsymbol{\zeta} \in \mathcal{M}^{2,4}$ is denoted by $[\boldsymbol{\zeta}]$. Thus, $\mathcal{N}$ defines a quotient space $\mathcal{N} / \sim \subset \mathbb{R} P^{5}$, denoted by $[\mathcal{N}]$ for convenience. It is obvious that $[\mathcal{N}]$ is homeomorphic to $S^{1} \times S^{3}$.

As well known, a general linear transformation on $\mathcal{M}^{2,4}$ induces a projective transformation on $\mathbb{R} P^{5}$. Since $\mathcal{N}$ is invariant under the $O(2,4)$ linear transformations ${ }^{1}$, they induce some transformations on $[\mathcal{N}]$. In $\$ 2.3$ we shall show how these transformations on $[\mathcal{N}]$ can be made into a conformal transformation on $[\mathcal{N}]$. In $\$ 3$ we shall show how these induced transformations on $[\mathcal{N}]$ can be viewed as "conformal transformations" on the Mink-space, $d S^{4}$ or $A d S^{4}$.

Before the topic of conformal transformations is concerned, we must investigate the problem of metric on $[\mathcal{N}]$. The metric $\boldsymbol{\eta}=\eta_{\hat{A} \hat{B}} d \zeta^{\hat{A}} \otimes d \zeta^{\hat{B}}$ on $\mathcal{M}^{2,4}$ cannot naturally induce a metric on $[\mathcal{N}]$. But it is not so bad.

A curve $\gamma$ in $\mathcal{N}$ can be projected to be a curve $[\gamma]$ in $[\mathcal{N}]$. However, the projection from $\gamma$ to $[\gamma]$ is not one-to-one. Another curve $\gamma^{\prime}$ in $\mathcal{N}$ can be projected to the same $[\gamma]$ in $[\mathcal{N}]$ iff their parameter equations differ from each other by a nonzero factor. We call such two curves in $\mathcal{N}$ are equivalent to each other. Given two equivalent curves $\zeta^{\hat{A}}=\zeta^{\hat{A}}(t)$ and $\zeta^{\hat{A}}=c(t) \zeta^{\hat{A}}(t)$ in $\mathcal{N}$, their line elements, $d s^{2}$ and $d s^{2}$, respectively, satisfy the relation

$$
d s^{2}=c^{2} d s^{2} .
$$

We can turn to the tangent spaces of $\mathcal{N}$ to formulate this result. We say that two tangent vectors, $\mathbf{X} \in T_{\zeta} \mathcal{N}$ and $\mathbf{X}^{\prime} \in T_{\zeta^{\prime}} \mathcal{N}$, are equivalent if $\left[\boldsymbol{\zeta}^{\prime}\right]=[\boldsymbol{\zeta}]$ and

\footnotetext{
${ }^{1}$ Strictly, $\mathcal{N}$ is invariant under the action of $O(2,4) \times \mathbb{R}$, where $r \in \mathbb{R}$ refers to a scale product on $\mathcal{M}^{2,4}$ by $e^{r}$. But the action of $\mathbb{R}$ induces the identity transformation on $\mathbb{R} P^{5}$. Thus it can be safely ruled out in our consideration.
} 
$\pi_{*} \mathbf{X}=\pi_{*} \mathbf{X}^{\prime}$ where $\pi_{*}$ is the pull-back of the natural projection $\pi: \mathcal{N} \rightarrow[\mathcal{N}]$. Now suppose $\mathbf{X}^{\prime}, \mathbf{Y}^{\prime} \in T_{\zeta^{\prime}} \mathcal{N}$ are equivalent to $\mathbf{X}, \mathbf{Y} \in T_{\zeta} \mathcal{N}$, respectively. Then,

$$
\boldsymbol{\eta}\left(\mathbf{X}^{\prime}, \mathbf{Y}^{\prime}\right)=c^{2} \boldsymbol{\eta}(\mathbf{X}, \mathbf{Y})
$$

where $c$ is the number in $\boldsymbol{\zeta}^{\prime}=c \boldsymbol{\zeta}$. This is the precise meaning that is implied, consciously or unconsciously, by eq. (4).

There are two important consequences of the above result. We describe them in $\$ 2.2$ and 2.3 respectively.

\subsection{Induced Metric and Weyl Transformations on $[\mathcal{N}]$}

It is not only that $[\mathcal{N}]$ is a quotient manifold, but also that all its tangent vectors can be viewed as residue classes: each residue class is a set of equivalent tangent vectors of $\mathcal{N}$. The usual way to deal with $[\mathcal{N}]$ is select a representative from each point in $[\mathcal{N}]$. If all the representatives are selected perfectly, we obtain an embedding $\phi:[\mathcal{N}] \rightarrow \mathcal{N}$ satisfying

$$
\pi \circ \phi=\operatorname{id}_{[\mathcal{N}]},
$$

where $\operatorname{id}_{[\mathcal{N}]}$ is the identity map on $[\mathcal{N}]$. Then the problem of selecting a representative for each tangent vector of $[\mathcal{N}]$ can be naturally solved by $\phi_{*}$. In this way we obtain a metric

$$
\mathrm{g}=\phi^{*} \boldsymbol{\eta}
$$

on $[\mathcal{N}]$. It is easy to see that $\mathbf{g}$ is a Lorentzian metric on $[\mathcal{N}]$.

If $\phi^{\prime}:[\mathcal{N}] \rightarrow \mathcal{N}$ is also an embedding satisfying $\pi \circ \phi^{\prime}=\operatorname{id}_{[\mathcal{N}]}$, then for any $[\boldsymbol{\zeta}] \in[\mathcal{N}]$, we have $[\boldsymbol{\zeta}]=\pi(\phi([\boldsymbol{\zeta}]))=\pi\left(\phi^{\prime}([\boldsymbol{\zeta}])\right)$. Thus there must be a nonzero real number $\Omega([\boldsymbol{\zeta}])$ so that

$$
\phi^{\prime}([\boldsymbol{\zeta}])=\Omega([\boldsymbol{\zeta}]) \phi([\boldsymbol{\zeta}]) .
$$

Therefore, the two embeddings $\phi$ and $\phi^{\prime}$ define a nonzero function $\Omega$ on $[\mathcal{N}]$. It is obvious that $\Omega$ is smooth.

Let $\mathbf{g}^{\prime}=\phi^{\prime *} \boldsymbol{\eta}$. Then it can be proved that

$$
\mathrm{g}^{\prime}=\Omega^{2} \mathbf{g} .
$$

That is, the consequence of the variation of embeddings is a Weyl transformation for the induced metric on $[\mathcal{N}]$.

\subsection{Conformal Transformations on $[\mathcal{N}]$}

In $\$ 2.1$ we have pointed out that an $O(2,4)$ transformation $O$ on $\mathcal{M}^{2,4}$ induces a transformation $[O]$ on $[\mathcal{N}]$, well defined by

$$
[O]([\zeta]):=[O \zeta]
$$


For a given $O \in O(2,4),[O]$ is a diffeomorphism on $[\mathcal{N}]$. Hence an action of $O(2,4)$ on $[\mathcal{N}]$ on the left is resulted in. However, such an action is not effective, because it can be easily verified that

$$
[-E]=[E]=\operatorname{id}_{[\mathcal{N}]}, \quad \text { or } \quad[-O]=[O]
$$

for arbitrary $O \in O(2,4)$, where $E$ is the identity transformation on $\mathcal{M}^{2,4}$. It can be proved that, for an $O \in O(2,4),[O]=\operatorname{id}_{[\mathcal{N}]}$ iff $O= \pm E$. It can be also proved that the action of $O(2,4)$ on $[\mathcal{N}]$ is transitive. So $[\mathcal{N}]$ is a homogeneous space of $O(2,4)$.

Let $\phi:[\mathcal{N}] \rightarrow \mathcal{N}$ be an embedding as described in 2.2 and $O$ be an $O(2,4)$ linear transformation. For an arbitrary $[\boldsymbol{\zeta}] \in[\mathcal{N}]$, we can set

$$
\boldsymbol{\zeta}:=\phi([\zeta]), \quad \boldsymbol{\zeta}^{\prime}:=\phi\left(\left[\boldsymbol{\zeta}^{\prime}\right]\right)=\phi([O \zeta]),
$$

which are contained in $\phi([\mathcal{N}]) \subset \mathcal{N}$ and can be treated as representatives of $[\boldsymbol{\zeta}]$ and $[O][\boldsymbol{\zeta}]$, respectively. On the other hand, since $\left[\boldsymbol{\zeta}^{\prime}\right]=\pi\left(\boldsymbol{\zeta}^{\prime}\right)=(\pi \circ \phi)([O \boldsymbol{\zeta}])=$ $\operatorname{id}_{[\mathcal{N}]}([O \boldsymbol{\zeta}])=[O \boldsymbol{\zeta}]$, there must be a nonzero real number $\rho([\boldsymbol{\zeta}])$, depending on $[\zeta]$, such that

$$
\boldsymbol{\zeta}^{\prime}=\rho([\zeta]) O \zeta .
$$

In this way we obtain a nonzero function $\rho$ on $[\mathcal{N}]$.

Now let $\mathbf{g}$ be the metric on $[\mathcal{N}]$ induced by the embedding $\phi:[\mathcal{N}] \rightarrow \mathcal{N}$, as shown in 2.2 It can be proved that $[O]$ is a conformal transformation:

$$
[O]^{*} \mathbf{g}=\rho^{2} \mathbf{g} \text {. }
$$

So, every $O(2,4)$ linear transformation on $\mathcal{M}^{2,4}$ induces a conformal transformation on $([\mathcal{N}], \mathbf{g})$. Due to eqs. (11), the conformal group of $([\mathcal{N}], \mathbf{g})$ is the quotient group $O(2,4) / \mathbb{Z}_{2}$.

\section{Conformal Transformations on the Mink-Space, $d S^{4}$ and $A d S^{4}$}

In $\$ 2.2$ and $\$ 2.3$ the representatives are selected in a perfect way that they form a submanifold $\phi([\mathcal{N}])$ diffeomorphic to $[\mathcal{N}]$. In this section we use a not so perfect method: only most of, but not all, points in $[\mathcal{N}]$ can find their respective representatives, located in a hyperplane $\mathcal{P}$ of $\mathcal{M}^{2,4}$ off $\boldsymbol{\zeta}=0$. The resulted space $\mathcal{P} \cap \mathcal{N}$ are Mink, $d S^{4}$ or $A d S^{4}$ according to whether the normal vector $\mathbf{n}$ of $\mathcal{P}$ is null, timelike or spacelike. And "on" these spaces there are the "conformal transformations" which are of great interest in physics.

\subsection{The Minkowskian Case}

When the normal vector $\mathbf{n}$ is null, it can be extended to be a linear basis $\left\{\mathbf{e}_{\mu}, \mathbf{n}, \mathbf{l}\right\}$ of $\mathcal{M}^{2,4}$, with $\mathbf{e}_{\mu}$ for $\mu=0, \ldots, 3$ tangent to $\mathcal{N}$ and $\mathcal{P}$, satisfying

$$
\mathbf{e}_{\mu} \cdot \mathbf{e}_{\nu}=\eta_{\mu \nu}, \quad \mathbf{e}_{\mu} \cdot \mathbf{n}=0, \quad \mathbf{e}_{\mu} \cdot \mathbf{l}=0, \quad \mathbf{l} \cdot \mathbf{l}=0, \quad \mathbf{n} \cdot \mathbf{l}=1 .
$$


It is easy to see that a point $\zeta \in \mathcal{P} \cap \mathcal{N}$ iff

$$
\boldsymbol{\zeta}=x^{\mu} \mathbf{e}_{\mu}+x^{+} \mathbf{n}+R \mathbf{l}, \quad x^{+}=-\eta_{\mu \nu} x^{\mu} x^{\nu} /(2 R),
$$

with $R$ a constant. And it is easy to verify that $\mathcal{N} \cap \mathcal{P}$ is a Mink-space because the induced metric on it is

$$
d s_{M}^{2}=\eta_{\mu \nu} d x^{\mu} d x^{\nu} .
$$

Now let us consider two equivalent curves with line elements $d \chi^{2}$ and $d s_{M}^{2}$, respectively. Assume that the former is just in $\mathcal{N}$, while the latter is in $\mathcal{P} \cap \mathcal{N}$. Then a relation similar to (44) can be obtained:

$$
d \chi^{2}=(\mathbf{n} \cdot \boldsymbol{\zeta} / R)^{2} d s_{M}^{2},
$$

where $\zeta$ is the point along the former curve. From eq. (18) it can be derived that the $O(2,4)$ linear transformations induce the so-called "conformal transformations" on the Mink-space 2].

\subsection{The $d S^{4}$ and $A d S^{4}$ Cases}

When the normal vector $\mathbf{n}$ is timelike, the induced metric on $\mathcal{P}$ has a signature as $\operatorname{diag}(1,-1,-1,-1,-1)$. Assume $\mathbf{n} \cdot \mathbf{n}=1$ and extend it to be an orthonormal basis $\left\{\mathbf{e}_{A}, \mathbf{n} \mid A=0,1, \ldots, 4\right\}$ of $\mathcal{M}^{2,4}$. Then $\boldsymbol{\zeta} \in \mathcal{P} \cap \mathcal{N}$ iff

$$
\boldsymbol{\zeta}=\xi^{A} \mathbf{e}_{A}+R \mathbf{n}, \quad \eta_{A B} \xi^{A} \xi^{B}=-R^{2},
$$

where $R$ is a positive constant and $\left(\eta_{A B}\right)=\operatorname{diag}(1,-1,-1,-1,-1)$. (We have carefully chosen $\mathbf{n}$ in order that $R>0$.) Thus $\mathcal{N} \cap \mathcal{P}$ is a $d S^{4}$ of radius $R$.

Let $d \chi^{2}$ and $d s_{+}^{2}$ be the line elements of two equivalent curves $\gamma$ and $\gamma_{+}$, respectively. Again the former is just in $\mathcal{N}$ and the latter is in $\mathcal{P} \cap \mathcal{N}$. Then, with $\zeta$ the point along $\gamma$, there is similarly the relation

$$
d \chi^{2}=(\mathbf{n} \cdot \boldsymbol{\zeta} / R)^{2} d s_{+}^{2} .
$$

Given an $O(2,4)$ linear transformation, $\gamma$ can be transformed to be another curve $\gamma^{\prime}$, lying still in $\mathcal{N}$ and equivalent to a curve $\gamma_{+}^{\prime}$ lying in $\mathcal{P} \cap \mathcal{N}$. Let their line elements be $d \chi^{\prime 2}$ and $d s_{+}^{\prime 2}$, respectively. Then a similar relation to (20) holds for $d \chi^{\prime 2}$ and $d s_{+}^{\prime 2}$. The $O(2,4)$ transformation preserves the line elements: $d \chi^{\prime 2}=d \chi^{2}$. Thus there will be

$$
d s_{+}^{\prime 2}=\left(\frac{\mathbf{n} \cdot \boldsymbol{\zeta}}{\mathbf{n} \cdot \zeta^{\prime}}\right)^{2} d s_{+}^{2}
$$

for $\gamma_{+}$and $\gamma_{+}^{\prime}$, where $\zeta$ and $\boldsymbol{\zeta}^{\prime}$ are a pair of equivalent points along $\gamma$ and $\gamma^{\prime}$, respectively. Therefore, similar to the Minkowskian case, an $O(2,4)$ linear transformation induces a "conformal transformation" on $d S^{4}$. 
In general a set of Beltrami coordinates 3, 4, 5] can be assigned to an equivalence class $[\boldsymbol{\zeta}]$. For $\boldsymbol{\zeta}$ as in eq. (19). The Beltrami coordinates for $[\boldsymbol{\zeta}]$ is

$$
x^{\mu}:=R \xi^{\mu} / \xi^{4}, \quad(\mu=0,1,2,3),
$$

provided that $\xi^{4} \neq 0$. In this coordinate system

$$
d s_{+}^{2}=\left[\frac{\eta_{\mu \nu}}{\sigma_{+}(x)}+\frac{\eta_{\mu \alpha} \eta_{\nu \beta} x^{\alpha} x^{\beta}}{R^{2} \sigma_{+}(x)^{2}}\right] d x^{\mu} d x^{\nu}, \quad \sigma_{ \pm}(x):=1 \mp R^{-2} \eta_{\mu \nu} x^{\mu} x^{\nu} .
$$

Here $\sigma_{-}(x)$ is preserved for $A d S^{4}$. The Beltrami coordinates must satisfy $\sigma_{+}(x)>0$ [4]. Isometries have the generic form as below [4, 5]:

$$
x^{\prime \mu}= \pm \frac{\sqrt{\sigma_{+}(a)} D_{\nu}^{\mu}\left(x^{\nu}-a^{\nu}\right)}{\sigma_{+}(a, x)}, \quad D_{\nu}^{\mu}:=L_{\nu}^{\mu}+\frac{L_{\alpha}^{\mu} \eta_{\nu \beta} a^{\alpha} a^{\beta}}{R^{2} \sqrt{\sigma_{+}(a)}\left(1+\sqrt{\sigma_{+}(a)}\right)}
$$

where $L=\left(L_{\nu}^{\mu}\right) \in O(1,3), \pm 1=\operatorname{det} L$ and the constants $a^{\mu}$ satisfy $\sigma_{+}(a)>0$. In the above, $\sigma_{ \pm}(a, x):=1 \mp R^{-2} \eta_{\mu \nu} a^{\mu} x^{\nu}$, where $\sigma_{-}(a, x)$ is preserved for $A d S^{4}$. Other conformal transformations include

$$
x^{\prime \mu}=\frac{x^{\mu} \sqrt{1-\beta^{2}}}{1 \pm \beta \sqrt{\sigma_{+}(x)}}, \quad(|\beta|<1)
$$

and

$$
x^{\prime \mu}=x^{\mu}-\frac{1-\sigma_{+}(b, x)}{1+\sqrt{\sigma_{+}(b)}} b^{\mu} \pm b^{\mu} \sqrt{\sigma_{+}(x)},
$$

where \pm corresponds to the coordinate neighborhoods where $\xi^{4}>0$ or $\xi^{4}<0$.

When $\mathbf{n}$ is spacelike, it can be similarly proved that $\mathcal{N} \cap \mathcal{P}$ is $A d S^{4}$, Similarly, $O(2,4)$ transformations induce conformal transformations. Beltrami coordinates can be also introduced in the same way as on $A d S^{4}$, and the conformal transformations take a similar form as in the above.

\section{The Extension of $A d S / C F T$ Correspondence}

\subsection{The Geometric Picture of $A d S /$ CFT Correspondence}

The discussion in 2 and 3 reveals a wonderful geometric picture as follows. The 4-d space $[\mathcal{N}] \cong S^{1} \times S^{3}$ is a hypersurface of $\mathbb{R} P^{5}$. Although no natural metric can be inherited from $\mathcal{M}^{2,4},[\mathcal{N}]$ can be realized (by an embedding $\phi$ as in $\$ 2.2]$ as a hypersurface $\phi([\mathcal{N}])$ of $\mathcal{N}$, enabling it to receive a metric $\mathbf{g}$ from the realization. The variousness of realizations ends up with Weyl transformations for the metric. Thus, $[\mathcal{N}]$ is rather a Weyl space than a spacetime, having a vanishing Weyl tensor. Hence theory of physics in $[\mathcal{N}]$, if exists, should be Weyl-invariant - at least it should be conformally invariant.

If the infinity boundary is included in the Mink-space, $d S^{4}$ and $A d S^{4}$, they are also a realization of $[\mathcal{N}]$, as if the projective plane model for $\mathbb{R} P^{2}$. 
What soever speaking, the Mink-space, $d S^{4}$ and $A d S^{4}$ can be embedded into $\mathcal{N}$, as shown in 3 . These three kinds of spaces, together with the perfect realizations of $[\mathcal{N}]$ as in $\$ 2$ can be related to each other by the projection. The maps from one to another are conformal maps, among which those from a Mink $/ d S^{4} / A d S^{4}$ to a Mink/d $S^{4} / A d S^{4}$ are of special interest, which will be discussed elsewhere [6].

Using the above conformal maps, a CFT on the Mink-space can be transferred to be CFTs on both $d S^{4}$ and $A d S^{4}$, and vice versa. This fact can be summarized as the conformal triality of Mink-, $d S$ - and $A d S$-spaces. In fact, a CFT on any of these spaces is a CFT on $([\mathcal{N}], \mathbf{g})$.

Topologically $A d S^{5}$ can be viewed as an open region in $\mathbb{R} P^{5}$, consisting of timelike 1 -d linear subspaces of $\mathcal{M}^{2,4}$. In this sense $[\mathcal{N}]=\partial\left(A d S^{5}\right)$. If the $A d S / C F T$ correspondence [1] is correct, then we can say that the corresponding CFT is on the Mink-space, on $d S^{4}$, on $A d S^{4}$, on $([\mathcal{N}], \mathbf{g})$. Thus we might have as many $A d S / C F T$ correspondences as possible.

The $A d S / C F T$ correspondence for higher dimensions can be also conjectured in the similar geometric picture.

\subsection{Null Geodesics}

As we know, up to re-parameterizations, null geodesics are invariant under conformal transformations and Weyl transformations. The null geodesics can also be illustrated in a geometric picture.

Suppose $\left[\boldsymbol{\zeta}_{0}\right]$ and $\left[\boldsymbol{\zeta}_{1}\right]$ are two distinct points in $[\mathcal{N}]$. Then $\boldsymbol{\zeta}_{0}$ and $\boldsymbol{\zeta}_{1}$ are two linearly independent null vectors, spanning a 2 -d linear subspace (plane) $\Sigma$ in $\mathcal{M}^{2,4}$. If, in addition,

$$
\zeta_{0} \cdot \zeta_{1}=0
$$

then the whole $\Sigma$ except the origin 0 is contained in $\mathcal{N}$. Thus $\Sigma \cap \mathcal{P} \subset \mathcal{N} \cap \mathcal{P}$, no matter whether the latter is the Mink-, $d S$ - or $A d S$-space. Obviously, $\Sigma \cap \mathcal{P}$ is a null straight line. If, in addition, we assume that $\boldsymbol{\zeta}_{0}$ and $\boldsymbol{\zeta}_{1} \in \mathcal{P}$, then the equation of $\Sigma \cap \mathcal{P}$ reads

$$
\zeta(\lambda)=(1-\lambda) \zeta_{0}+\lambda \boldsymbol{\zeta}_{1}
$$

For the 2-d linear subspace $\Sigma \subset \mathcal{M}^{2,4}$, an antisymmetric tensor

$$
\omega:=\zeta_{0} \otimes \zeta_{1}-\zeta_{0} \otimes \zeta_{1}
$$

can be defined in terms of its linear basis $\left\{\boldsymbol{\zeta}_{0}, \boldsymbol{\zeta}_{1}\right\}$. If the linear basis of $\Sigma$ is

changed, then the antisymmetric tensor $\boldsymbol{\omega}^{\prime}$ corresponding to the new basis is proportional to $\boldsymbol{\omega}$. In fact, $\boldsymbol{\omega}$ can be treated to be something like the area 2 -form of $\Sigma$.

Meanwhile, for the straight line (28), a 6-d angular momentum tensor

$$
\mathcal{L}:=\zeta \otimes \frac{d \zeta}{d \lambda}-\zeta \otimes \frac{d \zeta}{d \lambda}
$$


can be defined. Substituting eq. (28) into the above, we find that the angular momentum is conserved:

$$
\mathcal{L}=\omega
$$

It is very intuitive and can be proved that, in the Mink, $d S$ and $A d S$ cases, $\Sigma \cap \mathcal{P}$ is a null geodesic. The 6-d angular momentum can be expressed in terms of the 4-d angular momentum and the 4-momentum of the massless particle.

In order to see what it looks like in the Minkowski or Beltrami coordinates, we consider two special cases. In the first case, $\mathcal{P}$ is a null hyperplane $\zeta^{-}=R$, where $\zeta^{ \pm}=\frac{1}{\sqrt{2}}\left( \pm \zeta^{4}+\zeta^{5}\right)$. Substitution of eq. (16) to (28) yields

$$
x^{\mu}=\left(1-\frac{\tau}{R}\right) x_{0}^{\mu}+\frac{\tau}{R} x_{1}^{\mu}, \quad \tau=R \frac{\lambda \zeta_{1}^{-}}{(1-\lambda) \zeta_{0}^{-}+\lambda \zeta_{1}^{-}} .
$$

Let's write the energy-momentum and angular momentum as

$$
P^{\mu}=m \frac{d x^{\mu}}{d \tau}, \quad L^{\mu \nu}=x^{\mu} P^{\nu}-x^{\nu} P^{\mu},
$$

respectively and formally introduce

$$
P^{+}=m \frac{d x^{+}}{d \tau}, \quad L^{\mu+}=-L^{+\mu}=x^{\mu} P^{+}-x^{+} P^{\mu},
$$

with $x^{+}$as shown in eqs. (16). Then it can be verified that

$$
\mathcal{L}^{\mu \nu}=\frac{R}{m} L^{\mu \nu}, \quad \mathcal{L}^{\mu+}=\frac{R}{m} L^{\mu+}, \quad \mathcal{L}^{\mu-}=-\frac{R^{2}}{m} P^{\mu}, \quad \mathcal{L}^{+-}=-\frac{R^{2}}{m} P^{+} .
$$

In the second case, we take $\mathcal{P}$ as $\zeta^{5}=R$. In the corresponding Beltrami coordinate system, the equation of $\Sigma \cap \mathcal{P}$ is still of the form (32), only that $\tau$ is no longer an affine parameter. However, with the momentum and angular momentum still defined as in (33), they are conserved quantities, and there will be

$$
\begin{aligned}
& \mathcal{L}^{\mu \nu}=\frac{\zeta_{0}^{4} \zeta_{1}^{4}}{m R} L^{\mu \nu}, \quad \mathcal{L}^{\mu 4}=-\frac{\zeta_{0}^{4} \zeta_{1}^{4}}{m} P^{\mu}, \\
& \mathcal{L}^{\mu 5}=\mp \frac{\zeta_{0}^{4} \zeta_{1}^{4}}{m} \eta^{\mu \nu} \sigma(x)^{\frac{3}{2}} g_{\nu \rho}(x) P^{\rho}, \\
& \mathcal{L}^{45}=\mp \frac{\eta_{\mu \nu} x^{\mu}}{\sqrt{\sigma(x)}} \frac{\zeta_{0}^{4} \zeta_{1}^{4}}{m R} P^{\nu} .
\end{aligned}
$$

In the above, $\mp$ is opposite to the sign of $\xi^{4}$. If the normal vector of $\mathcal{P}$ is spacelike, the results are similar to the above.

\section{Conclusion}

From the null cone $\mathcal{N} \subset \mathcal{M}^{2,4}$, we can construct the Mink-space, $d S^{4}$ and $A d S^{4}$ on which the induced action of $O(2,4)$ is conformal. When $\mathcal{M}^{2,4}$ is viewed 
as the homogeneous space of $\mathbb{R} P^{5},[\mathcal{N}]:=\mathcal{N} / \sim$ is the conformal (extension of the) Mink-, $d S$ - or $A d S$-space. Various metrics can be endowed on $[\mathcal{N}]$, differing from one another by Weyl transformations. For a given metric among them, the $O(2,4)$ transformations induce conformal transformations on $[\mathcal{N}]$. Since $[\mathcal{N}]$, the Mink-, $d S$ - and $A d S$-spaces are related by conformal maps, a CFT in one space results in a CFT in each of other spaces. Therefore the AdS/CFT correspondence could be extended to all these spaces. The same idea can be generalized to higher dimensions.

We have shown some evidence that the role of Beltrami coordinates on $d S / A d S$-spaces is similar to that of the Mink- coordinates. In fact, in the study of kinematics and dynamics on $d S / A d S$-spaces [4 [5, it is also revealed. The similarity is so strong that special relativity can be appealed for on $d S / A d S$ spaces [4] [5].

\section{Acknowledgments}

Part of the contents of this talk is based on the co-operation [6] of Profs. Z. Xu, C. G. Huang and Dr. Y. Tian. Many thanks are expressed to them. We are also pleasant to thank Profs. Q. K. Lu, S. K. Wang, K. Wu and X. C. Song for their helpful discussions. B. Zhou would like to express his special thanks to Nankai Institute of Mathematics for the accommodation, especially to Profs. C. Bai and W. Zhang.

\section{References}

[1] J. Maldacena, Adv. Theor. Math. Phys. 2 (1998) 231, hep-th/9711200

[2] R. Penrose and W. Rindler, Spinors and Space-Time: Volume 2, Spinor and Twistor Methods in Space-Time Geometry, Cambridge University Press, 1988.

[3] E. Beltrami, Opere Mat., 1 (1868) 374-405.

[4] H.-Y. Guo, C.-G. Huang, Z. Xu and B. Zhou, Mod. Phys. Lett. A19 (2004) 1701 , see also hep-th/0311156 H.-Y. Guo, C.-G. Huang, Z. Xu and B. Zhou, Phys. Lett. A 331 (2004) 1-7, hep-th/0403171 H.-Y. Guo, C.G. Huang, and B. Zhou, Europhys. Lett. 72[6] (2005) 1-7, hep-th/0404010 H.-Y. Guo, C.-G. Huang, Y. Tian, Z. Xu and B. Zhou, hep-th/0405137

[5] The original idea is due to K. H. Look (Q. K. Lu), "Why the Minkowski metric must be used?" (1970) unpublished. For earlier works, see, K. H. Look, C. L. Tsou (Z.L. Zou) and H. Y. Kuo (H.-Y. Guo), Acta Phys. Sinica 23 (1974) 225; Nature (Shanghai, Suppl.), Mod. Phys. 1 (1980) 97; H. Y. Kuo, Kexue Tongbao (Chinese Sci. Bull.) 22 (1977) 487 (all in Chinese); H. Y. Kuo, Proc. of the 2nd M. Grossmann Meeting on General 
Relativity, ed. by R. Ruffini, (North-Holland Pub. Amsterdam, 1982) 801; H.-Y. Guo, Nucl. Phys. B (Proc. Suppl.) 6 (1989) 381.

[6] H.-Y. Guo, B. Zhou, C.-G. Huang, Y. Tian and Z. Xu, in preparation; B. Zhou, H.-Y. Guo et al, in preparation. 